\title{
CORRIGENDUM
}

\section{Science with the Murchison Widefield Array: Phase I results and Phase II opportunities - Corrigendum}
A. P. Beardsley, M. Johnston-Hollitt, C. M. Trott, J. C. Pober, J. Morgan, D. Oberoi, D. L. Kaplan, C. R. Lynch, G. E. Anderson, P. I. McCauley, S. Croft, C. W. James, O. I. Wong, C. D. Tremblay, R. P. Norris, I. H. Cairns, C. J. Lonsdale, P. J. Hancock, B. M. Gaensler, N. D. R. Bhat, W. Li, N. Hurley-Walker, J. R. Callingham, N. Seymour, S. Yoshiura, R. C. Joseph, K. Takahashi, M. Sokolowski, J. C. A. Miller-Jones, J. V. Chauhan, I. Bojičić, M. D. Filipović, D. Leahy, H. Su, W. W. Tian, S. J. McSweeney, B. W. Meyers, S. Kitaeff, T. Vernstrom, G. Gürkan, G. Heald, M. Xue, C. J. Riseley, S. W. Duchesne, J. D. Bowman, D. C. Jacobs, B. Crosse, D. Emrich, T. M. O. Franzen, L. Horsley, D. Kenney, M. F. Morales, D. Pallot, K. Steele, S. J. Tingay, M. Walker, R. B. Wayth, A. Williams and C. Wu

doi: 10.1017/pasa.2019.41, published by Cambridge University Press, December 13, 2019

In Beardsley et al. (2019), the solid and dashed ovals indicating known supernova remnants and those detected in Hurley-Walker et al (2019b), respectively, are missing in the published version.

Correct figure:
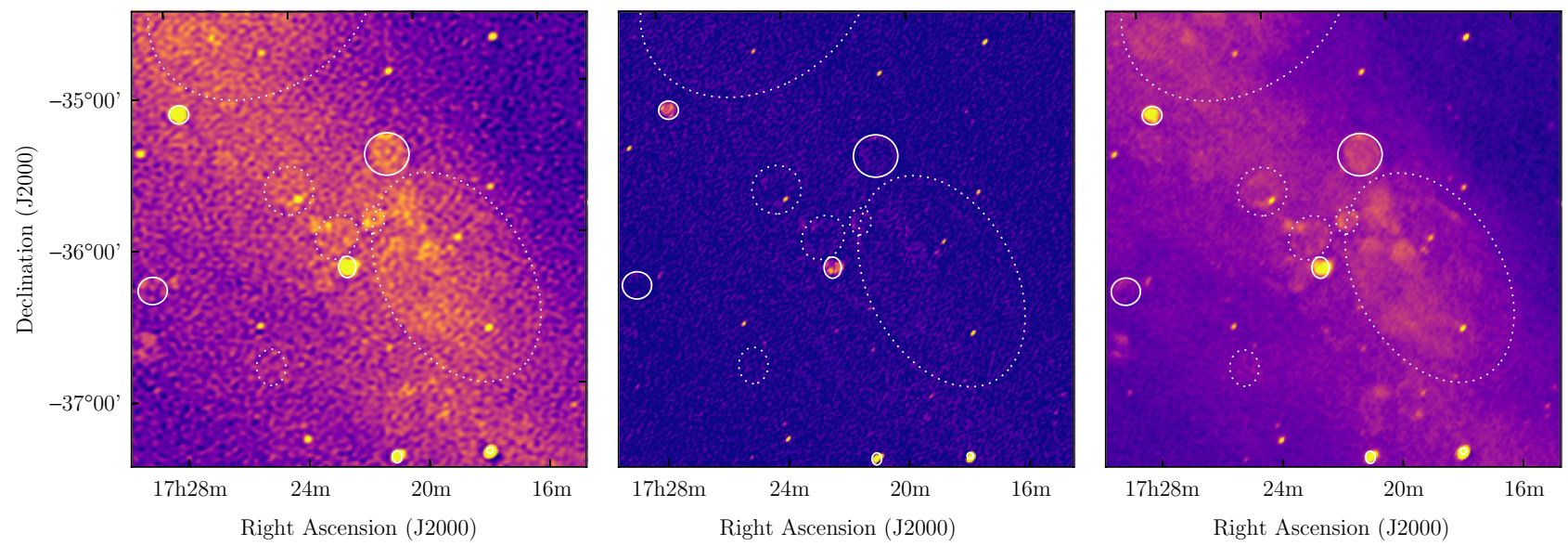

The authors apologize for this error.

\section{Reference}

Beardsley, A. P. et al. (2019). Science with the Murchison Widefield Array: Phase I results and Phase II opportunities. Publications of the Astronomical Society of Australia, 36, 1-30. doi: 10.1017/pasa.2019.41 